\title{
Desafios e Aplicações da Realidade Aumentada Móvel na Educação
}

\author{
Ezequiel Roberto Zorzal, ICT/Unifesp, ezorzal@gmail.com \\ Joaquim Armando Pires Jorge, IST/ULisboa, jorgej@tecnico.ulisboa.pt \\ Guilherme Guimarães Costa, ICT/Unifesp, guigcosta11@gmail.com
}

\begin{abstract}
Resumo: A Realidade Aumentada móvel pode ser aplicada em diversas áreas do conhecimento, dentre elas, podemos destacar o uso da tecnologia na educação. $\mathrm{O}$ objetivo deste artigo é apresentar uma análise das aplicações de Realidade Aumentada móvel para educação, considerando as áreas da educação que têm sido beneficiadas e o levantamento dos desafios da área. Foram analisados 83 trabalhos que apresentaram pesquisas com foco na aplicação da Realidade Aumentada móvel para educação. Percebemos que as aplicações relacionadas às áreas da biologia e história foram as mais abordadas nos trabalhos analisados, além disso, a Realidade Aumentada móvel foi utilizada na educação para melhorar a representação de objetos espaciais e simplificar os conceitos à eles associados, além de melhorar a colaboração e interação dos estudantes.
\end{abstract}

Palav ras-chave: Realidade Aumentada, Aprendizagem móvel, Educação, Ensino.

\section{Challenges and Applications of Mobile Augmented Reality in Education}

Abstract: The mobile Augmented Reality can be applied in several areas of knowledge, is possible to highlight the use of technology in education. The purpose of this article is to present an analysis of mobile Augmented Reality applications for education, considering the areas of education that have been benefited and the challenges of the area. We analyzed 83 papers that presented research focusing on the application of mobile Augmented Reality to education. We noticed that the applications related to the areas of biology and history were the most discussed in the papers analyzed. Furthermore, the Mobile Augmented Reality was used in education to improve the representation of spatial objects and to simplify the associated concepts, besides improving the collaboration and student interaction.

Keywords: Augmented Reality, Mobile learning, Education, Teaching.

\section{Introdução}

O uso dos telefones inteligentes (smartphones) tem sido indispensável para nosso cotidiano. Já sabemos que esta tecnologia vem alterando o comportamento do consumidor, ajudando os usuários a navegarem pelo mundo, alterando o modo como os consumidores fazem compras e ajudando os anunciantes a entrarem em contato com os clientes.

Em uma pesquisa realizada pela Digital, Social \& Mobile Worldwide (SOCIAL, 2018), mostrou que a utilização de dispositivos móveis vem aumentando constantemente. A pesquisa indicou que no ano de 2017 pelo menos $50 \%$ da população mundial teve acesso à Internet, $66 \%$ utiliza celulares e $34 \%$ da população possui contas ativas em redes sociais, o que representa um salto significativo em números relatados desde o relatório do ano anterior elaborado pela mesma empresa, quando a percentagem era apenas de $46 \%$ da população mundial com acesso à Internet, $51 \%$ possuía celulares e apenas $27 \%$ da população tinha contas ativas em redes sociais. Baseando-se nestas informações, o uso de celulares, em especial os smartphones, deverá impulsionar ainda mais o acesso à Internet da população mundial em pouco tempo. Além desse crescimento, a nomofobia, que é o desconforto ou a angústia causados pela incapacidade de comunicação através de aparelhos celulares ou computadores, tem se tornado comum entre as pessoas, principalmente entre os jovens e adolescentes 
(Caetano, 2017). Estas informações confirmam as preferências e um domínio cada vez maior do uso de dispositivos móveis no mundo.

Um dos principais benefícios desses dispositivos que pode ser citado é a possibilidade de adquirir conhecimento a partir da aprendizagem móvel (Mobile Learning). Já é sabido que este modelo de aprendizagem, se aplicado de forma correta, pode proporcionar inúmeras vantagens, tais como: mobilidade, engajamento, flexibilidade, rapidez e facilidade de acesso ao conteúdo. Com o avanço nas tecnologias de hardware, experiências computacionais, que anteriormente não poderiam ser executadas facilmente em dispositivos convencionais, tornaram-se comuns e já podem ser usadas amplamente no cotidiano, inclusive para apoiar o aprendizado.

Este avanço tecnológico, concomitante com a evolução científica, propiciaram a viabilização da Realidade Aumentada móvel. A Realidade Aumentada móvel consiste em complementar o ambiente real com informações virtuais, gerando um ambiente misturado. Para que isso ocorra, é necessário a utilização de algum dispositivo tecnológico que permita a visualização e a mobilidade do usuário em tempo real (Chatzopoulos et al., 2017). Em suma, os sistemas de Realidade Aumentada móveis consideram os meios de entrada de dados dos dispositivos, tais como câmera, giroscópio, microfones e GPS (Global Positioning System) para captar os dados que serão utilizados no processamento. Depois de processados, são registrados no ambiente real e apresentados na tela do dispositivo móvel. Desta maneira, por meio desta tecnologia, é possível utilizar o próprio dispositivo móvel do usuário para visualizar e interagir com os complementos virtuais gerados no ambiente misturado.

O uso da Realidade Aumentada móvel aplicada na educação vem sendo alvo de pesquisas no últimos anos. Diversos trabalhos estão sendo desenvolvidos com o objetivo de implementar sistemas de visualização com Realidade Aumentada móvel para fornecer interfaces acessíveis e de fácil utilização que apoiam o aprendizado e apresentam informações relevantes aos usuários.

Podemos citar, como exemplos, alguns trabalhos encontrados no repositório da RENOTE (Revista Novas Tecnologias na Educação): MACEDO et al. (2016) apresentam o desenvolvimento do aplicativo AppiRAmide para o estudo de geometria espacial para alunos do ensino médio e consideraram que os resultados obtidos foram satisfatórios; Da Silveira et al. (2011) utilizou a Realidade Aumentada em tablets para apresentar informações virtuais adicionais no Museu de Arte do Rio Grande do Sul Ado Malagoli para um grupo de pessoas; Galvão e Zorzal (2012) desenvolveram dois estudos de caso que envolveu o uso da Realidade Aumentada móvel para visualizar informações em livros infantis e para apoiar o ensino de superfícies de revolução na disciplina de geometria analítica; Panegalli et al. (2015) descreve a Realidade Aumentada como uma forma viável de estimular os estudantes apresentando uma aplicação para estudo das cores na língua inglesa; por fim, Herpich et al. (2017) usa a Realidade Aumentada móvel para auxiliar estudantes do ensino fundamental no processo de ensino-aprendizagem de geografia.

Apesar da constatação de existirem vários trabalhos contemplados na área da Realidade Aumentada móvel com enfoque na educação, não foram encontrados na literatura, trabalhos que forneçam uma visão geral do ponto de vista das áreas de aplicação e os desafios abordados nestes trabalhos. Assim, o objetivo deste artigo é tecer um panorama geral das aplicações de Realidade Aumentada móvel na educação, classificando-as por áreas de aplicação, assim como apresentar os desafios e, consequentemente, as oportunidades de pesquisa.

O artigo está organizado em quatro seções, além desta Introdução. Na Seção 2 é feita a apresentação dos critérios de pesquisa para a classificação das aplicações. A Seção 3 descreve os resultados e discussões, apresentando uma análise geral da pesquisa e os apontamentos dos principais desafios da área e a Seção 4 apresenta as considerações finais. 


\section{Metodologia}

Com a finalidade de analisar trabalhos correlatos à área de Realidade Aumentada móvel aplicada à educação e mapear os principais desafios remanescentes, a revisão deste trabalho tomou como base os artigos publicados nos últimos dez anos e que estão disponíveis na Web em diferentes bibliotecas digitais. Uma revisão da literatura foi realizada utilizando os seguintes repositórios de dados: ACM Digital Library, IEEE Xplore Digital Library e Springer Link .

Para a seleção dos trabalhos, foram utilizados os motores de busca das próprias bibliotecas. Após algumas buscas com palavras-chave relacionadas à pesquisa, entendemos que os motores de buscas foram mais eficientes ao definirmos como critérios a seguinte condição: Augmented Reality AND (Mobile OR Smartphone) AND (Education OR Learning OR Teaching). A lista dos repositórios, bem como as respectivas strings de busca que foram utilizadas neste trabalho podem ser visualizadas na Tabela 1.

Tabela 1. Lista dos repositórios e suas respectivas strings de busca.

\begin{tabular}{|l|l|c|c|}
\hline Repositório & String de busca & $\begin{array}{l}\text { Resultado da } \\
\text { busca }\end{array}$ & $\begin{array}{l}\text { Artigos } \\
\text { analisados } \\
\text { após filtros }\end{array}$ \\
\hline IEEE Xplore & $\begin{array}{l}\text { (("Document Title":Augmented Reality AND } \\
\text { "Document Title":mobile) AND ( "Document } \\
\text { Title":learning OR "Document Title":education OR } \\
\text { "Document Title":teaching)) }\end{array}$ & 37 & 26 \\
\hline $\begin{array}{l}\text { ACM Digital } \\
\text { Library }\end{array}$ & $\begin{array}{l}\text { acmdlTitle:(+augmented +reality +Mobile } \\
\text { education learning teaching) }\end{array}$ & 238 & 22 \\
\hline Springer Link & $\begin{array}{l}\text { Where the title contains: } \\
\text { "augmented reality" and mobile and ("education" } \\
\text { or "learning" or "teaching") }\end{array}$ & 687 & 35 \\
\hline
\end{tabular}

Assim, buscamos os trabalhos publicados que apresentaram em seus títulos ou palavras-chave o resultado da condição de busca elaborada para esta pesquisa. Atentamos para os artigos publicados a partir de Janeiro de 2008, ano subsequente ao lançamento da primeira geração do iPhone que aconteceu em Junho de 2007. Consideramos que a partir deste lançamento ocorreram grandes evoluções tanto no hardware quanto no software dos dispositivos móveis, tornando-os mais acessíveis aos usuários. Inclusive, podemos citar alguns trabalhos que foram desenvolvidos em tempos anteriores, tais como os trabalhos de Cheok et al. (2003) e Tamura et al. (2001) que apresentaram sistemas de Realidade Aumentada móvel baseados em computadores pessoais. A ideia inicial era reunir estes aparatos em combinação com tecnologias móveis em uma mochila para facilitar a mobilidade do usuário no ambiente. Embora interessante, o desconforto, tamanho e o peso dos equipamentos eram alguns fatores limitantes da proposta. Outras abordagens vieram com as propostas do uso de PDAs (Personal Digital Assistant) (Barakonyi e Schalstieg, 2006) e UMPCs (Ultra Mobile Personal Computer) (Peternier et al. 2007). Apesar de apresentarem resultados melhores, os PDAs e UMPCs eram uma alternativa com maior custo e dificultavam o uso da Realidade Aumentada móvel pela limitação de hardware. Logo, optamos por revisar os trabalhos mais recentes.

Para esta revisão, foram estabelecidos critérios de pesquisa e analisados os trabalhos correlatos em relação as suas características e funcionalidades, de acordo com o método abordado por Biolchini (2007). A revisão seguiu um protocolo pré-definido e foi dividida em etapas, conforme ilustrado na Figura 1. Isto garantiu a qualidade dos resultados obtidos no decorrer do processo. 


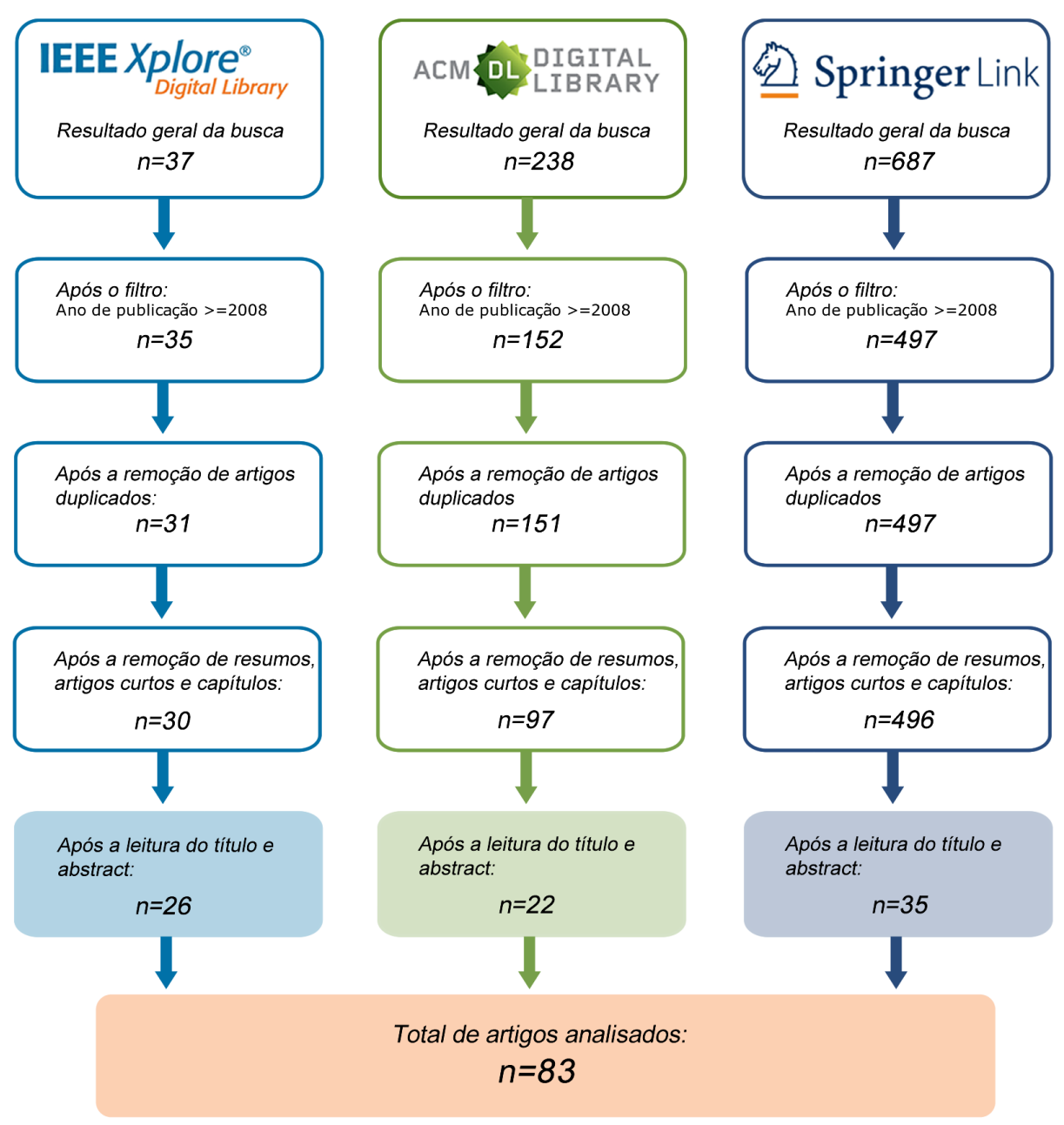

Figura 1. Etapas para a revisão dos artigos.

Foram analisados os títulos de todos os artigos encontrados e quando houve dúvida se o artigo seria considerado dentro da área correlata, seus resumos e textos foram analisados. Para que um artigo fosse selecionado para leitura completa deveria contemplar revisão, desenvolvimento ou reflexão sobre aplicações de Realidade Aumentada móvel na educação. Artigos resumidos ou referentes à capítulos de livros foram descartados. Conforme apresentado na Figura 1, inicialmente, foram encontrados o total de 962 publicações, no entanto, após os filtros estabelecidos no protocolo da pesquisa, foram selecionados 83 artigos que apresentaram as pesquisas focadas em aplicações de Realidade Aumentada móvel na educação (a lista dos artigos selecionados pode ser encontrada no seguinte sítio eletrônico: http://realidadeaumentada.org/data/RenoteZORZAL2018.pdf).

\section{Resultados e Discussões}

Os dados demográficos obtidos pela pesquisa podem ser verificados na Figura 2. Como pode ser notado na Figura 2(a), a quantidade numérica dos trabalhos que aplicam Realidade Aumentada móvel na educação vem aumentando gradualmente nos últimos anos. Cabe ressaltar que o gráfico da Figura 2 (a), no ano de 2018, apresenta apenas os artigos selecionados e analisados que estavam disponíveis nos repositórios de busca até o mês de Fev./2018.

Aproximadamente $43 \%$ dos trabalhos analisados foram desenvolvidos por pesquisadores oriundos de três países (Figura 2 (b)). Estados Unidos da América se 
destaca no desenvolvimento das aplicações correlatas por possuir a autoria maior que $18 \%$ dos trabalhos analisados. Taiwan e Espanha representam juntos um pouco mais de $23 \%$ das autorias dos trabalhos analisados.

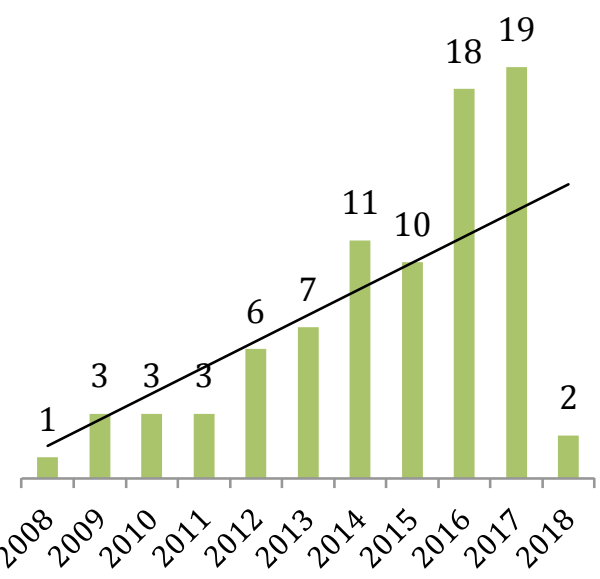

(a)

Figura 2. Análise geral dos trabalhos encontrados e que foram publicados nos últimos 10 anos. (a) Quantitativo dos trabalhos de Realidade Aumentada móvel aplicada na educação referente ao ano da publicação. (b) Percentual dos trabalhos referente aos países das instituições dos autores.

Também cabe mencionar que os trabalhos analisados foram desenvolvidos por diversas instituições localizadas em aproximadamente 27 países. Em alguns trabalhos pode-se apontar a ocorrência de alguma colaboração entre duas ou mais instituições distintas, como por exemplo, o trabalho de Nagata et al. (2017) que foi desenvolvido em conjunto por grupos de pesquisa da Universidade de Salamanca na Espanha e a Universidade Metropolitana de Ciências da Educação no Chile. Neste trabalho, os autores utilizaram a Realidade Aumentada móvel e mapas digitais para determinar a percepção dos estudantes sobre os processos educacionais, por meio de um aplicativo que ensina conceitos históricos e culturais. Os autores apresentaram bons resultados mostrando a efetividade de tais ferramentas. Em um contexto geral, o processo de colaboração entre grupos de pesquisas e instituições distintas pode acarretar em diversos benefícios, tais como o aumento da capacidade em resolver problemas e a melhoria das múltiplas competências.

Os trabalhos analisados também foram classificados em função da finalidade da área de especialização. As aplicações para o ensino são amplas e apresentam vários ramos específicos na educação, sendo assim, a Figura 3 apresenta as principais áreas de especialização identificadas nos trabalhos analisados. Muitos trabalhos que foram analisados abordaram apenas o conceito e processo pedagógico da utilização e métodos de visualização da Realidade Aumentada em ambientes de aprendizagem móvel e não apresentaram especificadamente a área de especialização estudada.

As aplicações relacionadas às áreas da biologia e história foram as mais abordadas nos trabalhos analisados. Juntas, abrangeram quase um terço de todas aplicações estudadas. No entanto, foi observado o uso da Realidade Aumentada móvel nas mais diversas áreas para o ensino. Podemos citar a aplicação para o público infantil desenvolvida por Rusiñol et al. (2017) para abstrair conceitos musicais, como a notação musical e o ritmo; e a aplicação da Realidade Aumentada móvel combinada com mapas conceituais para ensinar crianças com autismo a se socializar com outras pessoas (Lee et al., 2018). 
Acredita-se que os estudos de caso mais abordados nos artigos analisados estão diretamente relacionados às diferentes formas em que a Realidade Aumentada móvel pode ser aplicada e seus benefícios à ela associados. Foram identificados diferentes formatos de aplicação da Realidade Aumentada móvel de acordo com suas principais características. Percebeu-se que a Realidade Aumentada móvel foi utilizada na educação para melhorar a representação de objetos espaciais e simplificar os conceitos à eles associados, além de melhorar a colaboração e interação dos estudantes em locais físicos.

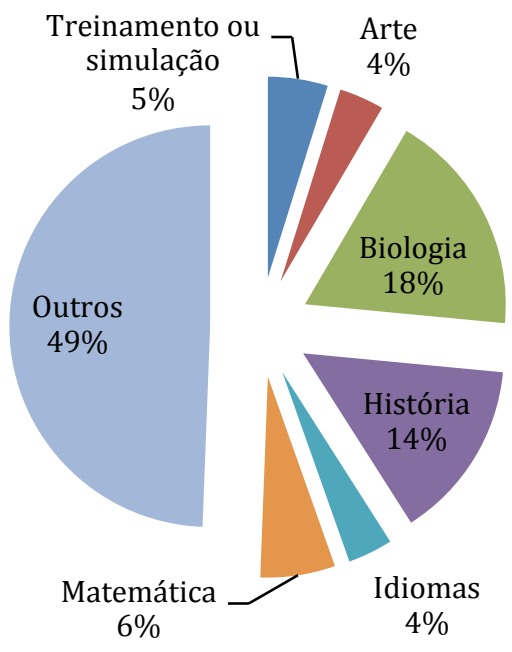

Figura 3. Classificação dos trabalhos analisados em função da área de especialização.

Alguns trabalhos especializados em história, por exemplo, mostraram que o uso da Realidade Aumentada móvel pode melhorar o aprendizado e a percepção dos estudantes ao apresentar elementos contextuais e informativos relevantes em ambientes históricos de acordo com a localização do estudante (Nilsson et al., 2012; Harley et al. 2016). O uso da Realidade Aumentada móvel permitiu associar as representações visuais com o ambiente natural de onde se originou os dados. $\mathrm{O}$ uso dos dispositivos móveis equipados com o GPS pode melhorar a interação entre os estudantes com os locais físicos em que as informações virtuais estão associadas.

Por outro lado, a motivação da maioria dos trabalhos relacionados à biologia foi o uso da representação tridimensional para acrescentar uma nova dimensão à representação dos dados e melhorar a percepção do espaço de visualização (Angeles et al., 2017; Karagozlu, 2017). Nestes trabalhos a Realidade Aumentada foi utilizada para melhorar a compreensão das informações no ambiente a partir de representações tridimensionais. Pode-se citar o trabalho de De Ravé et al. (2016) que consistiu em desenvolver um sistema para facilitar o aprendizado de geometria. $\mathrm{O}$ sistema mostrouse eficiente, gerando um impacto positivo na habilidade espacial dos estudantes, enquanto a aplicação provou-se capaz de ser executada adequadamente em diversos dispositivos, o que faz do sistema uma ferramenta flexível, de baixo custo e eficiente.

Alguns trabalhos focaram o desenvolvimento de conteúdo para o aluno ou o professor. Pode-se citar o trabalho de Sánchez et al. (2014) que apresenta um framework para gravar pontos de interesse em vários pontos históricos, mas que ainda apresenta limitações para criar cenários específicos para cada usuário.

Cabe mencionar que, ao analisar os trabalhos selecionados para esta pesquisa, notou-se que aproximadamente $41 \%$ destes trabalhos apresentaram alguma menção de agradecimento ao apoio financeiro parcial ou total da pesquisa. Uma hipótese é que aproximadamente $60 \%$ dos trabalhos analisados foram desenvolvidos de forma privada, sem qualquer auxílio financeiro por empresas ou órgãos de fomento. 
Vários desafios foram identificados nos trabalhos analisados. Apesar da existência de várias pesquisas e aplicações da Realidade Aumentada móvel na educação, a inserção efetiva desta tecnologia ainda é considerada um desafio, devido à dificuldade de integrá-las com os métodos de aprendizagem tradicionais. $\mathrm{O}$ desenvolvimento de aplicações e técnicas eficazes que engaja e prenda a atenção do aluno, por períodos duradouros, ainda é uma barreira para a área. Técnicas de gamificação já foram estudadas e implementadas para apoiar o desenvolvimento destas aplicações, os resultados mostraram-se motivadores mas ainda precisam ser aperfeiçoados (Lan, 2013; Laine et al., 2016).

O tamanho da tela do dispositivo móvel, precisão na localização do usuário, qualidade da câmera de vídeo, baixa velocidade na transmissão de dados, falta de memória e a gerência de grande volume de informações podem ser mencionados como alguns dos principais desafios técnicos encontrados ao se desenvolver aplicações para Realidade Aumentada móvel para a educação.

Outro desafio técnico discutido está relacionado ao problema de registro dos objetos virtuais inseridos ao ambiente real. O problema do registro diz respeito à necessidade de alinhar de maneira precisa os objetos virtuais que serão sobrepostos aos objetos reais. Em algumas aplicações essa exigência se faz necessária para garantir uma experiência adequada e efetiva com o usuário.

Os cuidados relacionados à saúde e desconforto dos usuários (Arvanitis et al., 2009), também é um desafio, quando se trata de aplicações que exigem principalmente a utilização de aparatos não convencionais, como a utilização de capacetes HMD (HeadMounted Display).

Também é necessário que as aplicações ofereçam características realistas. O ideal é que as proporções de tamanho e localização dos objetos no mundo virtual sejam observadas a fim de que representem com fidelidade o mundo real. Além disso, o desenvolvimento de experiências convincentes e realmente efetivas é que poderão fazer a diferença entre a utilização da Realidade Aumentada móvel na educação sob um aspecto de modismo ou algo que realmente faça sentido.

Em alguns trabalhos não foram identificadas avaliações com a participação efetiva de profissionais da área da educação. Nota-se que há uma necessidade de realizar avaliações, acompanhamentos e estudos de casos longitudinais para a validação das características gerais das aplicações em termos de eficácia, eficiência e satisfação, não apenas no que diz respeito ao funcionamento do produto e à conformidade com os padrões de usabilidade, mas também acerca das opiniões dos usuários sobre as suas explorações no sistema.

\section{Conclusões}

Conforme declarado no relatório técnico internacional NMC Horizon Report de 2017, a aprendizagem móvel está mudando a maneira como as pessoas interagem com o conteúdo e seus arredores. Alunos podem acessar materiais didáticos em qualquer lugar e a qualquer hora. Esta demanda gera novas oportunidades, com potencial para criação de materiais aprimorados para dispositivos móveis que podem aumentar o acesso à educação. Inclusive, os instrutores já estão aproveitando as capacidades dos dispositivos móveis para promover abordagens de aprendizagem criando novas oportunidades para os alunos se conectarem com o conteúdo do curso. Os dispositivos móveis estão provendo mais oportunidades para estudantes e instrutores interagirem (Becker et al., 2017).

A Realidade Aumentada móvel é uma tecnologia em expansão, ela tem contribuído de maneira significativa na área educacional. A aprendizagem móvel com técnicas de Realidade Aumentada permite associar as representações visuais com o ambiente natural de onde se originou os dados. Ela garante um grande potencial no desenvolvimento de aplicações educacionais, permitindo uma interação natural de fácil 
adaptação e livre de dispositivos especiais. Aplicações com Realidade Aumentada móvel possibilitam a mobilidade dos usuários e permitem que eles tenham uma visão enriquecida e ampliada do ambiente. Ao lidar com os objetos virtuais tridimensionais sobrepostos ao cenário real, percebeu-se que os estudantes podem estimular sua capacidade de percepção e raciocínio espacial (Lee et al., 2012; De Ravé et al., 2016, Martínez-Granã, et al., 2016; Salinas e González-Mendívil, 2017).

Aplicações de Realidade Aumentada móvel na educação apresentam um potencial muito grande de desenvolvimento. A área de trabalhos colaborativos, especificamente, apresenta um espaço interessante de desenvolvimento, em função da existência de múltiplos usuários interagindo em um mesmo espaço compartilhado. Esses ambientes exigirão novas formas de interação, gerando novas interfaces que deverão facilitar o trabalho das pessoas e o seu desenvolvimento cognitivo.

Por outro lado, é importante ressaltar que nem sempre a melhor solução é o uso de ambientes tridimensionais para a visualização de dados em ambientes educacionais. Em algumas situações, as informações a serem visualizadas são tão simples que uma apresentação com tais recursos pode comprometer o bom entendimento das informações e dificultar o aprendizado (Zorzal, 2009). Porem, as aplicações de Realidade Aumentada móvel na educação apresentam grandes potenciais e podem amplificar as capacidades dos estudantes avaliarem informações tridimensionais.

Realidade Aumentada móvel e interfaces inteligentes para a educação parecem ser uma boa possibilidade para o desenvolvimento de novas aplicações na área. Tais aplicações serão capazes de se adaptar a diferentes tipos de perfís dos estudantes, fazendo com que os mesmos possam reorganizar os módulos apresentados na interface da forma que achar mais agradável para seu uso. O ideal é fazer com que estas aplicações se adaptem aos objetivos dos estudantes e apresentem informações integradas e compreensíveis utilizando vários modos de comunicação.

As tecnologias com aprendizado adaptativo possibilitam monitorar o progresso do aluno, usando dados para modificar a instrução a qualquer momento. Além disso, permitem ajustar dinamicamente o tipo ou nível do conteúdo do curso, baseando-se nas habilidades do aluno (Becker et al., 2017).

Embora os trabalhos analisados tenham sido desenvolvidos, na maioria, por pesquisadores estrangeiros, recentemente no Brasil, têm surgido grupos de pesquisa que têm trabalhado com Realidade Aumentada móvel na educação.

\section{Agradecimentos}

Os autores agradecem à Fundação de Amparo à Pesquisa do Estado de São Paulo (FAPESP), processo $\mathrm{n}^{\circ}$ 2018/20358-0, pelo apoio à esta pesquisa.

\section{Referências Bibliográficas}

ANGElES, J. M., CALANDA, F. B., BAYON-ON, T. V. V., MORCO, R. C., AVESTRO, J., e CORPUZ, M. J. S. AR Plants. In Proceedings of the 2017 International Conference on Computer Science and Artificial Intelligence - CSAI 2017. ACM Press. 2017.

ARVANitis, T. N., PETROU, A., KNIGHT, J. F., SAVAS, S., SOTIRIOU, S., GARGALAKOS, M., e GIALOURI, E. Human factors and qualitative pedagogical evaluation of a mobile augmented reality system for science education used by learners with physical disabilities. Personal and Ubiquitous Computing, 13(3), 243-250. 2009.

BARAKONYI, I. e SCHMALSTIEG, D. (2006). "Ubiquitous animated agents for augmented reality," in Proceedings of the 5th IEEE and ACM International Symposium 
on Mixed and Augmented Reality, pp. 145-154, IEEE Computer Society, 2006.

BECKER, S.A., CUMMINS, M., DAVIS, A., FREEMAN, A., HALL GIESINGER, C., e ANANTHANARAYANAN, V. (2017). NMC Horizon Report: 2017 Higher Education Edition. Austin, Texas: The New Media Consortium. 2017.

BIOLCHINI, J.C. de A., MIAN, P.G., NATALI, A.C., CONTE, T.U. e TRAVASSOS, G.H. 2007. Scientific research ontology to support systematic review in software engineering. Adv. Eng. Inform. 21, 2 (April 2007), 133-151.

CAETANO, M. B. Crise de atenção ou nomofobia - os desafios da educação na adolescência, Unoesc \& Ciência-ACHS, vol. 8, no. 1, pp. 37-50, 2017.

CHATZOPOUlOS, D., BERMEJO, C., HUANG , Z., e HUI, P. Mobile augmented reality survey: From where we are to where we go. IEEE Access, vol. 5, pp. 69176950, 2017.

CHEOK, A., FONG, S., GOH, K., YANG, X., LIU, W., FARZBIZ, F. e Li, Y. Human pacman: A mobile entertainment system with ubiquitous computing and tangible interaction over a wide outdoor área. Human-computer interaction with mobile devices and services, pp. 209-223, 2003.

DA SILVEIRA, A.L.M., BIAZUS, M.C.V., e AXT, M. Realidade Aumentada no Margs: Impressões de um Experimento. RENOTE 9.2. 2011.

DE RAVÉ, E. G., JIMÉNEZ-HORNERO, F. J., ARIZA-VILLAVERDE, A. B., e TAGUAS-RUIZ, J. DiedricAR: a mobile augmented reality system designed for the ubiquitous descriptive geometry learning. Multimedia Tools and Applications, 75(16), 9641-9663. 2016.

GALVAO, M.A., e ZORZAL, E.R. Aplicações Móveis com Realidade Aumentada para Potencializar Livros. RENOTE 10.1 (2012).

HARLEY, J. M., POITRAS, E. G., JARRELL, A., DUFFY, M. C., e LAJOIE, S. P. Comparing virtual and location-based augmented reality mobile learning: emotions and learning outcomes. Educational Technology Research and Development, 64(3), 359388. 2016.

HERPICH, F., NUNES, F. B., VOSS, G. B., SINDEAUX, P., TAROUCO, L. M. R., e DE LIMA, J. V. Realidade Aumentada em Geografia: uma atividade de orientação no ensino fundamental. RENOTE, 15(2), 2017.

KARAGOZLU, D. Determination of the impact of augmented reality application on the success and problem-solving skills of students. Quality \& Quantity. 2017.

LAINE, T. H., NYGREN, E., DIRIN, A., e SUK, H.-J. Science Spots AR: a platform for science learning games with augmented reality. Educational Technology Research and Development, 64(3), 507-531. 2016.

LAN, T. S. Learning through augmented reality mobile game application. In 2013 IEEE 63rd Annual Conference International Council for Education Media (ICEM). IEEE. 2013.

LEE, I.-J., CHEN, C.-H., WANG, C.-P., e CHUNG, C.-H. Augmented Reality Plus Concept Map Technique to Teach Children with ASD to Use Social Cues When Meeting and Greeting. The Asia-Pacific Education Researcher, 27(3), 227-243. 2018. 
LEE, K. Augmented Reality in Education and Training. TechTrends, 56(2), 13-21. 2012.

MACEDO, A. DE C., DA SILVA, J.A. e BURIOL, T.M., Usando Smartphone e Realidade aumentada para estudar Geometria espacial. RENOTE, 14(2). 2016.

MARTÍNEZ-GRAÑA, A. M., LEGOINHA, P., GONZÁLEZ-DELGADO, J. A., DABRIO, C. J., PAIS, J., GOY, J. L., e CUNHA, T. Augmented Reality in a Hiking Tour of the Miocene Geoheritage of the Central Algarve Cliffs (Portugal). Geoheritage, 9(1), 121-131. 2016.

NAGATA, J. J., GINER, J. G.-B., e ABAD, F. M. Augmented reality and pedestrian navigation through of mobile implementation on heritage content, in Proceedings of the Fourth International Conference on Technological Ecosystems for Enhancing Multiculturality - TEEM '16, 2016.

NILSSON, S., ARVOLA, M., SZCZEPANSKI, A., e BÅNG, M. (2012). Exploring place and direction. In Proceedings of the 24th Australian Computer-Human Interaction Conference on - OzCHI '12. ACM Press. 2012.

PANEGALli, F.S., CAGLIARI, D.C., BERNARDI, G., CORDENONSI, A.Z. e MALLMANN, E.M., Realidade Aumentada no Desenvolvimento de Jogos Educacionais: um Estudo de Caso de um Jogo de Língua Inglesa. RENOTE, 13(1). 2015.

PETERNIER, A., RIGHTTI, X., HOPMANN, M., THALMANN, D., REPETTOY, M., PAPAGIANNAKIS, G., DAVY, P., LIM, M., MAGNENAT-THALMANN, N., BARSOCCHI, P., FRAGOPOULOS, T., SERPANOS, D., GI- ALELIS, Y. e KIRYKOU, A. Chloe@university: An indoor, mobile mixed reality guidance system. 2007 ACM Symposium on Virtual Reality Software and Technology, VRST '07, (New York, NY, USA), pp. 227-228, ACM, 2007.

RUSIÑOL, M., CHAZALON, J., e DIAZ-CHITO, K. Augmented songbook: an augmented reality educational application for raising music awareness. Multimedia Tools and Applications. 2017.

SALINAS, P., \& GONZÁLEZ-MENDÍVIL, E. (2017). Augmented reality and solids of revolution. International Journal on Interactive Design and Manufacturing (IJIDeM), 11(4), 829-837.

SÁNCHEZ, R. A., REDONDO, E., e FONSECA, D. Geo-located teaching using handheld augmented reality: good practices to improve the motivation and qualifications of architecture students. Universal Access in the Information Society, 14(3), 363-374. 2014.

SOCIAL, W.A., 2017. Digital in 2017: Global overview. Disponível em: $<$ https://www.slideshare.net/wearesocialsg/digital-in-2017-global-overview>. Acesso em: 07 mai. 2018.

TAMURA, H., YAMAMOTO, H. e KATAYAMA, A. (2001). "Mixed reality: Future dreams seen at the border between real and virtual worlds," IEEE Computer Graphics and Applications, vol. 21, no. 6, pp. 64-70, 2001.

ZORZAL, E.R. Estratégia para o desenvolvimento de aplicações adaptativas de visualização de informação com Realidade Aumentada. Tese (Doutorado) Universidade Federal de Uberlândia, Minas Gerais, 2009. 